\title{
Le relevé MAS, données 2017, démarre en novembre
}

\author{
Jacques Huguenina, Frédéric Clausen ${ }^{b}$ \\ a Dr, chef de section, section Services de santé, Office fédéral de la statistique, Neuchâtel

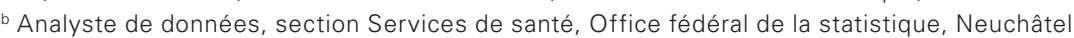

Le premier relevé des «données structurelles des cabinets médicaux et centres ambulatoires» (relevé MAS) s'est achevé avec succès mi-2017. Les premiers résultats ont été publiés début 2018. Le deuxième relevé MAS démarrera le 12 novembre 2018 et portera sur les données de l'année 2017. Dans cette perspective, l'e-questionnaire a été remanié et optimisé. Le relevé MAS est obligatoire pour tous les cabinets médicaux et centres ambulatoires de Suisse. Les données seront récoltées à des fins statistiques et de surveillance légale.

\section{Les premiers résultats du relevé MAS ont été publiés}

Le premier relevé des «données structurelles des cabinets médicaux et centres ambulatoires» (relevé MAS) a été réalisé de novembre 2016 à juin 2017. Il portait sur les données de l'année 2015. En tout, 18723 entreprises ont été interrogées, dont $47 \%$ ont livré les données. Le taux de livraison a varié entre $24 \%$ et $67 \%$ selon le canton. Une publication de l'Office fédéral de la statistique (OFS), parue en mars 2018, analyse en détail la population de base et les caractéristiques de la participation [1]. Les premiers résultats du relevé ont été publiés par l'OFS en avril 2018 [2].

Les données du premier relevé MAS sont mises à disposition de tiers par l'OFS dans le cadre de la loi sur la statistique fédérale (LSF). Seules des données anonymes (agrégées ou individuelles) sont transmises. Ainsi, l'identification d'un cabinet en particulier n'est pas possible. Toute transmission de données individuelles fait l'objet d'un contrat de protection des données.

\section{Le deuxième relevé MAS débutera le 12 novembre}

Le deuxième relevé MAS débutera le 12 novembre 2018 et durera jusqu'au 28 février 2019. Il portera sur les données de l'année 2017. Dès lors, le relevé MAS sera réalisé à un rythme annuel. Les données de l'année 2016 ne seront pas relevées. Pour soutenir les entreprises interrogées, une hotline sera mise en place par l'OFs pendant la période de relevé. Cette hotline sera désormais également atteignable sur le temps de midi. Pour rappel, le relevé MAS est obligatoire pour tous les cabinets médicaux et centres ambulatoires de Suisse.

\section{Situation juridique claire}

Les données 2017, comme les données 2015, seront relevées à des fins statistiques, conformément à la LSF ainsi qu'à l'art. 23 de la loi sur l'assurance-maladie (LAMal). Suivant les principes du traitement statistique de l'OFS, seules des données anonymes sont publiées. Les données 2017 seront également relevées à fins de surveillance légale. Le mandat légal de collecter des données à des fins de surveillance est inscrit à l'art. 59a LAMal. L'OFS a défini dans un règlement de traitement, conformément à l'art. 30c de l'ordonnance sur l'assurance-maladie (OAMal), quelles données peuvent être mises à disposition de quels destinataires à des fins de surveillance légale. Le règlement de traitement applicable aux données 2017 est entré en vigueur le 20 mars 2017. Les données des cabinets médicaux et centres ambulatoires transmises à des fins de surveillance légale sont anonymisées selon selon le règlement de traitement.

\section{E-questionnaire a été remanié et optimisé}

Dans la perspective du deuxième relevé MAS, l'OFS a remanié et optimisé l'e-questionnaire, tout en conservant inchangé son contenu (variables et définitions). 
Pour ce faire, l'OFs s'est appuyé sur les nombreux enseignements tirés du premier relevé et sur la coopération précieuse de nombreux partenaires comme la FMH, les associations professionnelles, les partenaires d'interfaces (FMH, MedReg, NewIndex y. c. CdC, RoKo), les TrustCenters et les fournisseurs de logiciels. L'e-questionnaire remanié a été testé par l'OFS et ses partenaires dans le cadre d'un pilote entre mai et juin 2018.

\section{Une étape "typologie» et un "question- naire court» ont été ajoutés}

Une nouvelle étape, nommée "typologie», a été ajoutée à l'e-questionnaire. Cette étape permet de caractériser le(s) type(s) d'activité de l'entreprise sur ses sites de prise en charge (points of care) et de l'orienter vers le questionnaire adapté à sa situation (questionnaire standard ou questionnaire court). Le questionnaire court, nouvellement ajouté, permettra de saisir la situation des entreprises dont le chiffre d'affaire annuel est inférieur ou égal à 30000 francs ou qui n'ont pas d'infrastructure propre $(10 \%$ des entreprises interrogées en 2015).

\section{La conclusion de l'e-questionnaire a été optimisée}

La conclusion de l'e-questionnaire a été simplifiée. De plus, une étape de conclusion supplémentaire permet à l'entreprise interrogée, par le biais de deux cases, de confirmer à quelles fins les données transmises pourront être utilisées. Par défaut, les deux cases sont cochées et les données transmises à des fins statistiques et de surveillance légale, conformément à la loi. L'entreprise interrogée conserve cependant la possibilité de décocher une des deux cases.

\section{Les interfaces ont été renforcées en collaboration avec les partenaires et l'ergonomie améliorée}

L'ergonomie de l'e-questionnaire et la navigation en son sein ont été retravaillées et améliorées de façon à ce que l'e-questionnaire puisse être rempli plus aisément. Des adaptations techniques rendent plus facile l'identification des champs qui restent à compléter. Une nouvelle fonctionnalité permet à l'entreprise interrogée d'afficher le contenu du questionnaire en fonction de son utilisation (but statistique ou de surveillance légale). Enfin, en collaboration avec les partenaires d'interfaces, les modalités d'accès aux interfaces NewIndex et RoKo ont été optimisées. Les produits LAMal peuvent désormais être importés. De plus, une interface pour les données de medkey est nouvellement disponible.

L'OFS remercie d'ores et déjà tous les cabinets médicaux et centres ambulatoires qui participeront au relevé MAS des données 2017. Des informations supplémentaires sur le relevé MAS 2017 sont disponibles sur le site Internet de l'OFS (www.mas-f.bfs.admin.ch).

\section{Références}

1 Creiser C. Analyse de la participation et de la population suite au premier relevé sur les données structurelles des cabinets médicaux et centres ambulatoires (MAS). Neuchâtel: Office fédéral de la statistique; 2018.

2 Clausen F. Les cabinets médicaux et centres ambulatoires en 2015. Neuchâtel: Office fédéral de la statistique; 2018. 